\title{
Editorials
}

\section{Rapid access to brief psychological treatments for self-harm and suicidal crisis}

\section{SUICIDE AND SELF-HARM ARE PUBLIC HEALTH ISSUES}

Suicide is an important public health issue. Over 800000 people die by suicide each year worldwide, ${ }^{1}$ and approximately 6000 people per year in the UK. ${ }^{2}$ Self-harm is a public health priority. ${ }^{3}$ Self-harm is defined as any intentional act of self-injury or self-poisoning regardless of motivation or suicidal intent. ${ }^{4}$ Self-harm is associated with personal and social difficulties, as well as increased risk of suicide and premature death by other causes. The number of presentations for suicidal crisis without physical injury at emergency departments is unknown. In the UK, the increasing impact of those visits is evident through the increasing number of community crisis resolution/home treatment/'first response' schemes, and more recently the Core 24 response initiative. ${ }^{5}$ The Core 24 response initiative involves the provision of care for people experiencing a mental health crisis who attend in emergency departments, available 24 hours a day, 7 days a week.

For self-harm, there are over 200000 presentations to emergency departments annually. ${ }^{4.6}$ This includes people who have physically harmed themselves without suicidal intent and those who have attempted to die by suicide. However, it is important to note that many people who self-harm do not present to hospital and may be managed within community settings. The true prevalence of self-harm or suicidal crisis is therefore estimated to be much higher.? There are potential lifelong implications of non-suicidal self-harm and suicide attempts, such as an increased frequency of suicide, especially if the behaviours are adopted as a long-term coping strategy. Worryingly, adolescents and young adults are highly vulnerable to finding themselves in a crisis and rates in these groups is increasing for both self-harm and suicide. 8

\section{WHAT IS A SUICIDAL CRISIS?}

A suicidal crisis is a temporary state that occurs in response to overwhelming distress, characterised by unbearable emotional pain of such intensity and severity that death by

\section{"Rapid access to interventions provides an opportunity to quickly bring together early help and support, which suggests positive outcomes.}

suicide seems to be the only solution. For some people this is a time when they may have had suicidal thoughts or attempted to take their own lives. In terms of selfharm, often a point where self-harm risk is high or self-harm has already happened. In all these cases, a time when intervention seems important and vital. Previous research supports this as emergency department data on repetition rates and suicide rates following attendance for initial self-harm, suicide attempts, or suicidal ideation are high. ${ }^{10}$

\section{INCREASING RAPID ACCESS TO TREATMENT}

Challenges exist for increasing access to rapid interventions as many people may not present to hospital services when in crisis. Often physical health takes precedence over psychological factors in a crisis context, at least where self-harm has already taken place. While there is evidence that psychological therapies can help reduce the risk of self-harm, ${ }^{11}$ waiting lists can be long, thus increasing the possible risk of deterioration. Effective suicide prevention and intervention is vital due to the prevalence of the problem.

Current initiatives addressing suicidal crisis or self-harm focus on mental health crisis in secondary care and are not acknowledging that most self-harm and suicidal crisis occurs in primary care or within community settings. Psychosocial assessment by trained mental health professionals is recommended in the National Institute for Health and Care Excellence (NICE) guidelines ${ }^{12}$ for self-harm and suicidal ideation, and includes the assessment of patients' needs and risks with the aim of determining appropriate aftercare. The psychosocial assessment includes an evaluation of mental, physical, and emotional health. The Department of Health ${ }^{3}$ reports that further action and strengthening of ongoing efforts in the implementation of key effective suicide prevention interventions is vital. Rapid access to interventions provides an opportunity to quickly bring together early help and support, which suggests positive outcomes. However, further research is needed to provide robust findings.

\section{EVERYONE'S BUSINESS}

Over recent years, there has been increased reporting of self-harm and suicidal ideation from teachers and charity based or thirdsector organisations in frontline contact with children and adolescents. ${ }^{9}$ In the UK, the National Suicide Prevention Strategy ${ }^{13}$ has expanded its scope by aiming to reduce self-harm rates as a common precursor to suicide. One recommendation being adopted across the country is a single point of access for all service users in crisis, 24 hours a day, as part of the assessment and immediate care service - Core $24 .{ }^{5}$ This ensures rapid response to a crisis, short-term input lfor example, by emergency department mental health liaison teams), and, whenever possible, other alternatives to admission. Most people in suicidal crisis or who may self-harm do not need admitting to hospital. A growing evidence base and service user feedback has highlighted the potential harmful consequences and more long-term negative outcomes of being admitted to hospital. However, effective alternative services are lacking, or there is little evidence known about services that have been implemented and are working well. Data on good practice models that can be replicated within other health and social care systems is needed. More research on the benefits of having accessible professionals, well developed community services, and specific policies for treating patients who present in suicidal crisis or following self-harm would be useful.

\section{TREATMENT OPTIONS}

There is some evidence that specifically structured psychological therapies can 


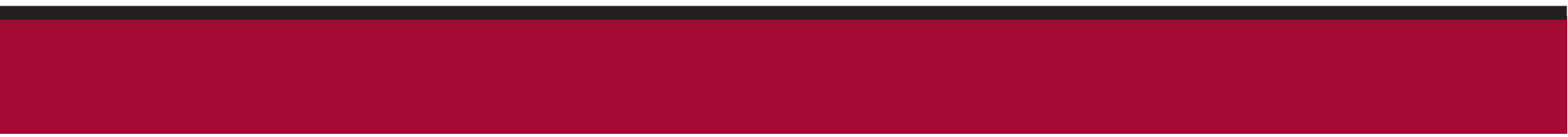

\section{"One recommendation being adopted across the country is a single point of access for all service users in crisis, 24 hours a day, as part of the assessment and immediate care service - Core 24.}

be effective in reducing repetition of selfharm or suicidality.1114,15 Recommendations suggest that interventions should be tailored to individual needs and should include cognitive-behavioural, psychodynamic, or problem-solving elements. Therapists are required to be trained and supervised in the therapy being offered and able to work collaboratively with the person to identify the problems causing suicidal distress or leading to self-harm. A barrier, however, is that some psychological services will not see people in a suicidal crisis or those who have recently self-harmed.

\section{IMPROVING ACCESS TO PSYCHOSOCIAL INTERVENTIONS}

While patients may contact the NHS for suicidal crisis or self-harm, they may not be referred to a specialist service that treats people in a crisis, specifically for these issues. Crisis resolution teams and home treatment teams offer biopsychosocial intervention, but this may not be universal. Often the focus will be on risk monitoring, medication management, and the ongoing review of medication side effects and adherence. Training for staff may not be available universally on delivering psychosocial interventions to people in crisis. Thus, some patients may not receive the psychosocial aspect of care alongside crisis risk management. Patients may be referred for psychological therapy; however, waiting lists can be up to months. Furthermore, a person may not meet the criteria for psychological services if they disclose selfharm or suicidal thoughts, leaving limited options for people who may find themselves in a crisis. The voluntary sector can also play a key role in providing support to people in crisis. However, little is known about its contribution within mental health crisis care ${ }^{16}$ and if the suicide prevention interventions are regulated, evidence informed, or follow a governance structure.

\section{IS RAPID ACCESS TO HELP IN THE MOMENT OF CRISIS EFFECTIVE?}

Rapid access to interventions at point of crisis is important and could help in reducing suicides. Brief psychological interventions have been shown to be effective in the prevention of suicide and self-harm. ${ }^{11,14,15}$ While some have reported promising findings, there remains a paucity of evaluative studies that considers the effectiveness of implementation of suicide prevention programmes. A recent pilot study reported reduced repetition of self-harm, reduced suicidality, and reduced readmission to hospital following rapid access to brief psychological therapy..$^{15}$ However, to date, research has been focused on emergency department data. More research is needed on rapid response services delivered within community settings to fully understand how effective they are.

\section{Pooja Saini}

Senior Lecturer, Liverpool John Moores University, Liverpool, UK

\section{REFERENCES}

1. World Health Organization (WHO). Suicide in the world: global health estimates. WHO, 2019

2. Office for National Statistics (ONS). Suicides in the UK: 2018 registrations. ONS, 2019.

3. Department of Health. Achieving better access to mental health services by 2020 . NHS England, 2019

4. Hawton K, Harriss L, Hall S, et al. Deliberate self-harm in Oxford, 1990-2000: a time of change in patient characteristics. Psychol Med

5. NHS England, National Collaborating Centre for Mental Health, National Institute for Health and Care Excellence. Achieving better access to 24/7 urgent and emergency mental health care - part 2: implementing the evidence-based treatment pathway for urgent and emergency liaison mental health services for adults and older adults - guidance. 2016.

6. Hawton K, Bergen H, Casey D, et al. Self-harm in England: a tale of three cities. Multicentre study of self-harm. Soc Psychiatry Psychiatr Epidemiol 2007; 42(7): 513-521.

7. Geulayov G, Kapur N, Turnbull P, et al. Epidemiology and trends in non-fatal self-harm in three centres in England, 2000-2012: findings from the Multicentre Study of Self-harm in England. BMJ Open 2016; 6: e010538.

8. Geulayov G, Casey D, McDonald KC, et al. Incidence of suicide, hospital-presenting nonfatal self-harm, and community-occurring nonfatal self-harm in adolescents in England lthe iceberg model of self-harm): a retrospective study. Lancet Psychiatry 2018; 5(2): 167-174.

9. McManus S, Gunnell D, Cooper C, et al. 2003; 33(6): 987-995.

\section{ADDRESS FOR CORRESPONDENCE}

\section{Pooja Saini}

Liverpool John Moores University, Tom Reilly

Building, Byrom Street, Liverpool L3 3AF, UK.

Email: P.Sainidljmu.ac.uk

\section{Cecil Kullu}

Consultant Psychiatrist, Mersey Care NHS Trust, Liverpool, UK.

\section{Emma Mullin}

HOPE Therapist, Mersey Care NHS Trust, Liverpool, UK

\section{Jane Boland}

Clinical Lead, James' Place Therapeutic Centre for Men in Suicidal Crisis, Liverpool, UK.

\section{Peter Taylor}

Clinical Senior Lecturer, University of Manchester, Manchester, UK

\section{Provenance}

Freely submitted; externally peer reviewed.

\section{Competing interests}

The authors have declared no competing interests.

DOI: https://doi.org/10.3399/bjgp20X709913

Prevalence of non-suicidal self-harm and service contact in England, 2000-14: repeated cross-sectional surveys of the general population. Lancet Psychiatry 2019; 6: 573-581.

10. The University of Manchester. National Confidential Inquiry into Suicide and Safety for Mental Health [NCISH]. Annual report 2019: England, Northern Ireland, Scotland and Wales. University of Manchester, 2020.

11. Hawton, K., Witt, K.G., Taylor Salisbury TL, et al. Psychosocial interventions for self-harm in adults. Cochrane Database Syst Rev.2016 12(5): CD012189.

12. National Institute for Health and Care Excellence (NICE). Self-Harm. QS34. NICE, 2013.

13. Department of Health and Social Care. Preventing suicide in England: Fourth progress report of the cross-government outcomes strategy to save lives. Department of Health and Social Care, 2019.

14. McCabe R, Garside R, Backhouse A, Xanthopoulou P. Effectiveness of brief psychological interventions for suicidal presentations: a systematic review. BMC psychiatry 2018; 18(1): 120.

15. Taylor PJ, Fien $\mathrm{K}$, Mullholland $\mathrm{H}$, et al. Pilot service evaluation of a brief psychological therapy for self-harm in an emergency department: Hospital Outpatient Psychotherapy Engagement (HOPE) service. Psychology Psychotherapy, Theory Research and Practice 2020; DOI:10.1111/papt.12277.

16. Newbigging K, Mohan J, Rees J. Harlock, et al. Contribution of the voluntary sector to mental health crisis care in England: protocol for a multimethod study. BMJ Open 2017; 7(11): e019238. 